\title{
AZD9291-resistant non-small cell lung cancer cell-derived exosomal Inc-MZT2A-5:1 induces the activation of fibroblasts
}

\author{
Liwei Song' ${ }^{1 \#}$, Gang Qian ${ }^{2 \#}$, Jia Huang', Tianxiang Chen ${ }^{1}$, Yunhai Yang ${ }^{1}$ \\ ${ }^{1}$ Shanghai Pulmonary Tumor Medical Center, Shanghai Chest Hospital, Shanghai, China; ${ }^{2}$ Department of Thoracic Surgery, Zhangjiagang Third \\ People's Hospital, Suzhou, China \\ Contributions: (I) Conception and design: Y Yang; (II) Administrative support: Y Yang, T Chen; (III) Provision of study materials or patients: L Song, \\ J Huang; (IV) Collection and assembly of data: L Song, G Qian; (V) Data analysis and interpretation: L Song; (VI) Manuscript writing: All authors; \\ (VII) Final approval of manuscript: All authors. \\ \#These two authors contributed equally to this work. \\ Correspondence to: Dr. Tianxiang Chen; Dr. Yunhai Yang. Shanghai Chest Hospital, Shanghai Pulmonary Tumor Medical Center, 241 West Huai-Hai \\ Road, Shanghai 200030, China. Email: zjutxchen@163.com; docyyh@163.com.
}

Background: AZD9291 resistance is still a challenge in the treatment of non-small cell lung cancer (NSCLC) and fibroblasts in the tumor microenvironment (TME) play a key role in the malignant phenotype of NSCLC. The study aimed to investigate the role of exosomes derived from AZD9291-resistant cells on the phenotypes of lung fibroblasts and the underlying mechanism.

Methods: The supernatants and exosomes of wild type and AZD9291-resistant NSCLC (H1975/PC9) cells were collected, and co-cultured with lung fibroblasts (MRC-5 cells) respectively. Transwell and quantitative real-time PCR (qRT-PCR) assays were used to evaluate migration and inflammation levels. Exosomes were collected by ultracentrifugation, and identified by nanoparticle tracking analysis (NTA), transmission electron microscopy (TEM) and western blots. Microarray was used to screen dysregulated exosomal lncRNAs from the resistant cells. Candidate lncRNAs were selected by bioinformatical annotation of their target genes and verified by qRT-PCR. The target lncRNA was then selected for further confirmation.

Results: Both the supernatant and exosomes from resistant cells significantly promoted the migration of MRC-5 cells, and the exosomes also upregulated mRNA levels of inflammation cytokines. Microarray identified 159 dysregulated exosomal lncRNAs. Fifteen candidate lncRNAs were selected following the biological roles of their target genes. qRT-PCR validation indicated that lnc-MZT2A-5:1 had the highest fold change. Finally, we found that lnc-MZT2A-5:1 could promote the migration ability and inflammation cytokines expression level of MRC-5 cells.

Conclusions: Our study clarified that lnc-MZT2A-5:1 from AZD9291-resistant NSCLC cell lines could promote the activation of MRC-5 cells, thus to uncover a new mechanism for AZD9291 resistance and provide new potential targets for the treatment of NSCLC.

Keywords: AZD9291 resistance; non-small cell lung cancer (NSCLC); exosome; lnc-MZT2A-5:1; fibroblasts

Submitted Aug 06, 2021. Accepted for publication Oct 22, 2021.

doi: $10.21037 / \mathrm{atm}-21-5186$

View this article at: https://dx.doi.org/10.21037/atm-21-5186

\section{Introduction}

Lung cancer is a leading cause of death worldwide, with 1.8 million people diagnosed and 1.6 million people dying from the disease every year (1). Non-small cell lung cancer (NSCLC), consisting of squamous cell carcinoma, large cell carcinoma and adenocarcinoma, is the predominant histologic subtype, accounting for approximately $85 \%$ of all lung cancer cases (2). The 5 -year overall survival (OS) rate of patients with NSCLC is about $17.8 \%$ (3).

The effective application of various targeted therapies 
and immunotherapy in some patients with advanced NSCLC has made great progress (4). Instead of traditional surgical interventions, targeted therapy and immunotherapy has changed the course of treatment for most patients, and molecular testing is now the standard recommendation for those with advanced lung adenocarcinoma (5). Osimertinib (AZD9291) is a third-generation tyrosine kinase inhibitors (TKIs) which selectively targets activating EGFR mutations and T790M-resistance mutation by formation of a covalent bond to the C797 residue of mutant EGFR (6). Despite the great success made by AZD9291 not only in first-line therapy but also in salvage therapy for T790M secondary mutations, acquired resistance inevitably emerges, limiting its long-term clinical benefits (7). However, the mechanism of drug resistance of AZD9291 is still not fully understood (8). Therefore, a better understanding of the drug resistance mechanism of targeted therapy and personalized treatment of lung cancer patients have become research hotspots in this field.

Exosomes range in diameter from 30 to $150 \mathrm{~nm}$ and are released into the extracellular environment by fusing with cell membranes (9). While at first, it was thought that exosomes only function when cells remove unnecessary molecules, many studies have subsequently shown they can transfer their contents (such as proteins and non-coding RNA) from one cell to another, thereby regulating cellular intercommunication $(10,11)$. Exosomes may participate in or interfere with the continuous crosstalk between cells or the local/distant host environment, thereby affecting tumor progression.

In normal tissue, fibroblasts are embedded in extracellular matrix (ECM), in an inactive quiescent state. While in tumor microenvironment (TME), fibroblasts are activated, termed as carcinoma-associated fibroblasts (CAFs) (12). Fibroblasts in the TME play a key role in the malignant phenotype of NSCLC (13). Recent studies have shown that exosomes are involved in regulating the sensitivity of tumor cells to chemotherapy drugs (14). Drug-resistant cancer cells can encapsulate chemotherapeutic agents in exosomes and transport anticancer drugs from tumor cells, which confers resistance to sensitive cells (15). In breast cancer cells, exosome-derived miR-221/222 could enhance the resistance of ER-positive breast cancer cells to tamoxifen, and exosomederived miR-522-3p could increase the resistance of PC9 cells $(16,17)$. Exosomes can also enhance drug resistance of tumor cells by inducing epithelial mesenchymal transition (EMT) or enhancing anti-apoptotic signaling pathways (18). However, the relationship between exosomes and lung cancer phenotypes has not yet been elucidated in AZD9291-resistant NSCLC cells.

In this study, we found that supernatant and exosomes derived from wild type or AZD9291-resistant H1975 and PC9 cells could promote the migration of MRC-5 cells. Moreover, we identified the differentially expressed lncRNAs in the exosomes by microarray analysis. Five candidate lncRNAs were then selected for further investigation according to the bioinformatics analysis of their predicted targets. qRT-PCR was performed to verify the expression levels of the five candidate lncRNAs, and we found that lnc-MZT2A-5:1 and lnc-CCDC103-7:1 were significantly increased in exosomes derived from AZD9291-treated H1975 and PC9 cells. Interestingly, we found that the overexpression of lnc-MZT2A-5:1 but not lnc-CCDC103-7:1 could significantly promote the migration and inflammation of MRC-5 cells. In summary, we identified new mechanisms of AZD9291 resistance and provided new potential targets for the treatment of NSCLC. We present the following article in accordance with the MDAR reporting checklist (available at https://dx.doi.org/10.21037/atm-21-5186).

\section{Methods}

\section{Cells and cell culture}

Human MRC-5 was purchased from the Cell Bank of the Chinese Academy of Sciences (Shanghai, China) and cultured in Minimum Essential Medium (MEM; Gibco, Carlsbad, USA) supplemented with $10 \%$ fetal bovine serum (Gibco, Carlsbad, USA). Human NSCLC cell lines PC9 and H1975 cells were obtained from the American Type Culture Collection (Manassas, VA, USA). The AZD9291resistant H1975 and PC9 cell line were established in a previous study (19), while NSCLC cell lines and the resistant cell lines were cultured in RPMI-1640 medium (Gibco, Carlsbad, USA) supplemented with 10\% FBS. All cells were incubated at $37{ }^{\circ} \mathrm{C}$ in a humidified atmosphere containing $5 \% \mathrm{CO}_{2}$.

\section{Migration assay}

For cell migration assays, a total of $1 \times 10^{5} \mathrm{MRC}-5$ cells in $100 \mu \mathrm{L}$ serum-free medium were seeded on transwell plates with inserts (Corning, USA). For detecting exosomes or cell supernatant function, equal quantities of supernatant or exosomes of wild type or AZD9291-resistant H1975/ PC9 cells were added into the inserts. We added $600 \mu \mathrm{L}$ complete medium into the bottom chambers, and the cell 
inserts were then incubated for $24 \mathrm{~h}$ and fixed and stained.

\section{Isolation and determination of exosomes}

Wild type or AZD9291-resistant H1975/PC9 cells were seeded in a $10 \mathrm{~cm}$ dish and cultured for $48 \mathrm{~h}$, then the supernatant collected and filtered. Exosomes in the supernatant were isolated by centrifugation. In brief, a total of $500 \mathrm{~mL}$ culture medium was centrifuged at $2,000 \times \mathrm{g}$ for $10 \mathrm{~min}$ at $4{ }^{\circ} \mathrm{C}$ to remove dead cells, and $10,000 \times \mathrm{g}$ for $30 \mathrm{~min}$ at $4^{\circ} \mathrm{C}$ to remove cell debris. Finally, exosomes were isolated by centrifugation at $110,000 \times \mathrm{g}$ for $90 \mathrm{~min}$ at $4{ }^{\circ} \mathrm{C}$. The exosomes were washed with PBS twice, and stored in PBS at $-80{ }^{\circ} \mathrm{C}$. The number and size distribution of exosomes were analyzed by nanoparticle tracking analysis (NTA), and microvesicles were detected by transmission electron microscopy (TEM).

\section{Western blot}

Cell total proteins were extracted by RIPA lysis buffer, and samples were separated by SDS-PAGE. Proteins were transferred onto a polyvinylidene fluoride membrane, which was incubated with primary antibody at $4{ }^{\circ} \mathrm{C}$ overnight. Protein expression was performed using an enhanced chemiluminescence kit (GE Healthcare, Pittsburgh, USA), while the density of the bands on blots was analyzed by Image $\mathrm{J}$ software (National Institutes of Health). The antibodies against CD81 (cat. no. ab109201), CD63 (cat. no. ab134045), HSP27 (cat. no. ab109376), and TSG101 (cat. no. ab125011) were from Abcam (Cambridge, UK).

\section{$R N A$ extraction and quantitative real-time PCR}

Trizol reagent (Invitrogen, Carlsbad, USA) was used to extract Total RNA from cells, which was then reverse transcribed into cDNAs using the Reverse Transcription kit (RiboBio, Guangzhou, China). Quantitative real-time PCR (qRT-PCR) was performed to amplify the cDNA template using a SYBR Premix Dimmer Eraser kit (RiboBio, Guangzhou, China) on the ABI 7500 system, and the relative mRNA levels of genes were determined by using the comparative $2^{-\Delta \Delta \mathrm{Ct}}$ method. All primer sequences are listed in Table S1.

\section{Microarray and bioinformatics analysis}

Exosomal lncRNAs microarray analysis was performed at the Shanghai Biotechnology Corporation (Shanghai, China), using Agilent Human miRNA 8*60K V21.0 microarray (Agilent Technologies, USA). Quantile normalization and subsequent data processing were performed using Quantile algorithm, Gene Spring Software 12.6 (Agilent Technologies). The expression of lncRNAs with a FC abs $\geq 2$ was considered significantly changed, and genes in the upstream and downstream of lncRNA within the range of $10 \mathrm{~kb}$ were selected as cis-target genes for lncRNAs. According to the prediction of trans-target genes, those genes having complementary or similar sequence with IncRNAs were selected, the complementary energy between the two sequences was then calculated by RNAplex, and genes with $e \leq-30$ were selected as the trans-target genes of lncRNAs. Go and KEGG analysis of predicted target genes were performed through DAVID (https://david.ncifcrf. gov/) (20).

\section{Cell transfection}

lnc-MZT2A-5:1, lnc-CCDC103-7:1, and their negative control (NC) were synthesized by RiboBio (Guangzhou, China), and equal amounts of each were transfected in MRC-5 cells according to the instructions of the Lipofectamine 2000 kit (Invitrogen, Carlsbad, USA). Cells were then harvested after 36 hours for analysis.

\section{Statistical analysis}

All data are presented as the mean $\pm \mathrm{SD}$. and were analyzed with SPSS version 22.0 (SPSS, Chicago, USA), and each experiment was performed at least three times (biological replicate). Differences between two groups or multiple groups were respectively performed with the Student's $t$-test or one-way ANOVA followed by Bonferroni post-hoc tests. Comparisons of data among multiple groups were performed by one way analysis of variance followed by Tukey's post hoc tests, and $\mathrm{P}<0.05$ was considered statistically significant.

\section{Results}

Supernatant of AZD9291-resistant NSCLC cells promoted
the migration of MRC-5 cells To detect the mechanism of AZD9291 resistance, we cocultured MRC-5 cells with the supernatant of NSCLC (H1975 and PC9 cells) sensitive or resistant to AZD9291. We found that the supernatant of wild type cells could 

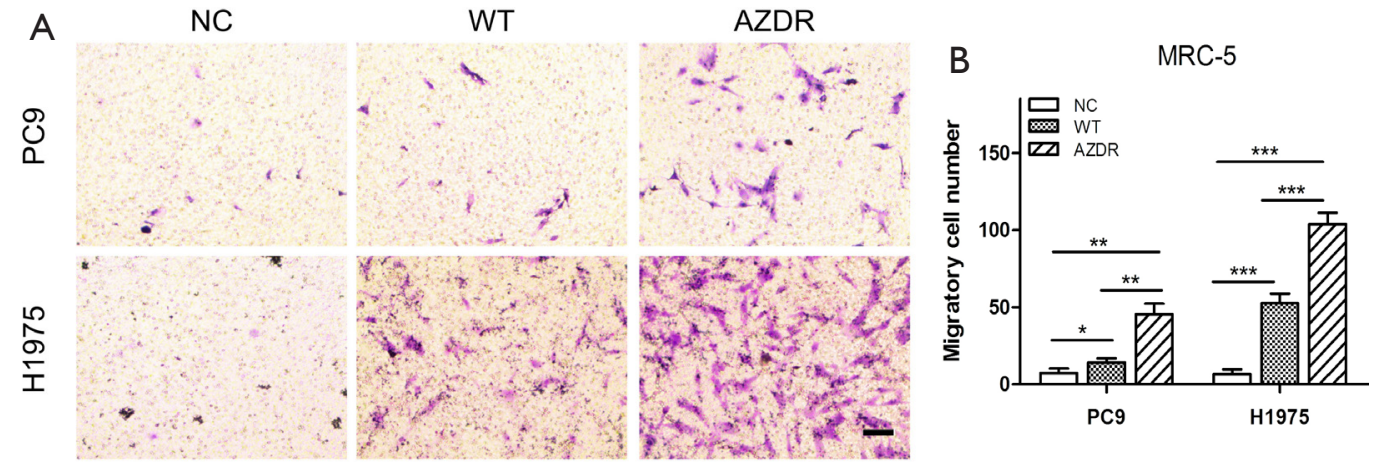

Figure 1 The supernatant of wild type and AZD9291-resistant H1975 and PC9 cells promoted the migration of MRC-5 cells. (A) Representative images of transwell assays analysis of MRC-5 cells co-cultured with or without supernatant of wild type or AZD9291resistant H1975/PC9 cells stained by crystal violet. (B) Statistical analysis of transwell assays ( $\mathrm{n}=3$ ). A length of $50 \mu \mathrm{m}$ was shown by scale bar and statistical significance was distinguished with $\mathrm{P}$ value: ${ }^{*}, \mathrm{P}<0.05 ;{ }^{* *}, \mathrm{P}<0.01$; ${ }^{* *}, \mathrm{P}<0.001$. NC, negative control; WT, wild type.
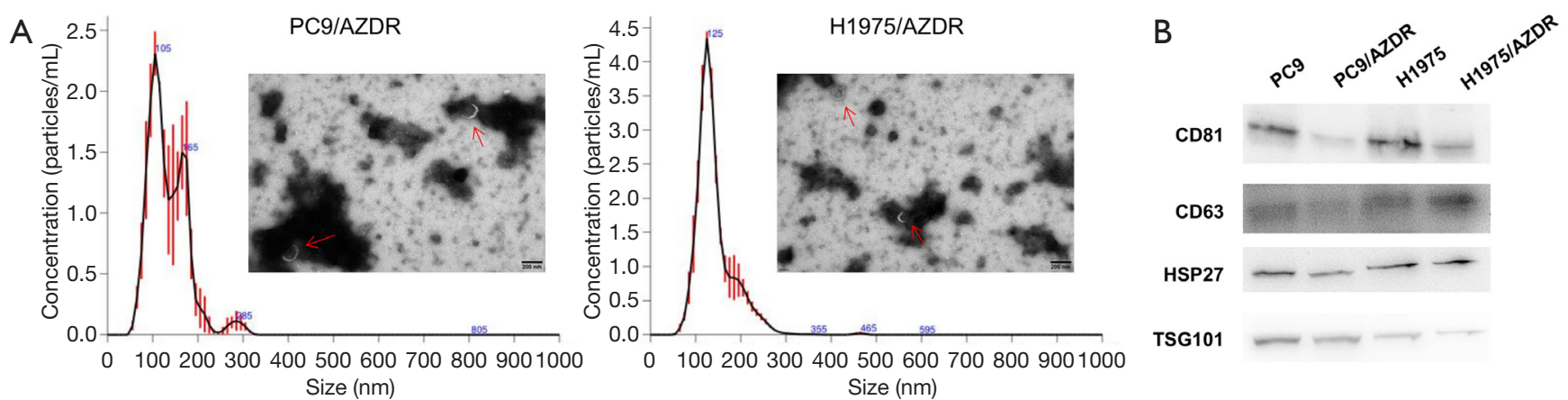

Figure 2 Characterization of exosomes derived from wild type or AZD9291-resistant H1975/PC9 cells. (A) NTA analysis of the size distribution, and TNM images of exosomes shown by the red arrows. (B) Western blotting analysis for exosomal markers (CD81, CD63, Hsp27, and TSG101) of exosomes. NTA, nanoparticle tracking analysis.

significantly promote the migration of MRC-5 cells compared to the $\mathrm{NC}$ group. while the supernatant from the resistant cells could further promote the migration of MRC-5 cells compared to the wild type group (Figure 1). Those data indicated that the AZD9291-resistant NSCLC cells could promote the migration of MRC- 5 cells by some secreted factors in the supernatant.

\section{Identification of exosomes derived from NSCLC cells}

To explore the mechanism underlying the regulation of supernatant of AZD9291-resistant NSCLC cells on the migration of MRC-5 cells, exosomes were isolated and purified from the supernatant of wild type and AZD9291resistant PC9/H1975 cells, respectively. The diameter of exosomes was detected by TEM and NTA, and all exosomes showed typical nanoparticles wrapped by lipid bilayer membranes. The size of these nanoparticles ranged from 50 to $200 \mathrm{~nm}$ (Figure 2A). In addition, we also analysed the protein markers of isolated exosomes by Western blot, and $\mathrm{s}$ shown in Figure 2B, the proteins of CD81, CD63, TSG101, and Hsp27 were positively expressed in all exosomes. These results indicate that we successfully isolated exosomes secreted by wild type and AZD9291-resistant NSCLC cells.

\section{Exosomes derived from wild type and AZD9291-resistant NSCLC cells promoted the migration and inflammation of MRC- 5 cells}

To explore the biological effects of exosomes derived from wild type and AZD9291-resistant NSCLC cells on MRC-5 cells, we next co-cultured MRC-5 cells with or 

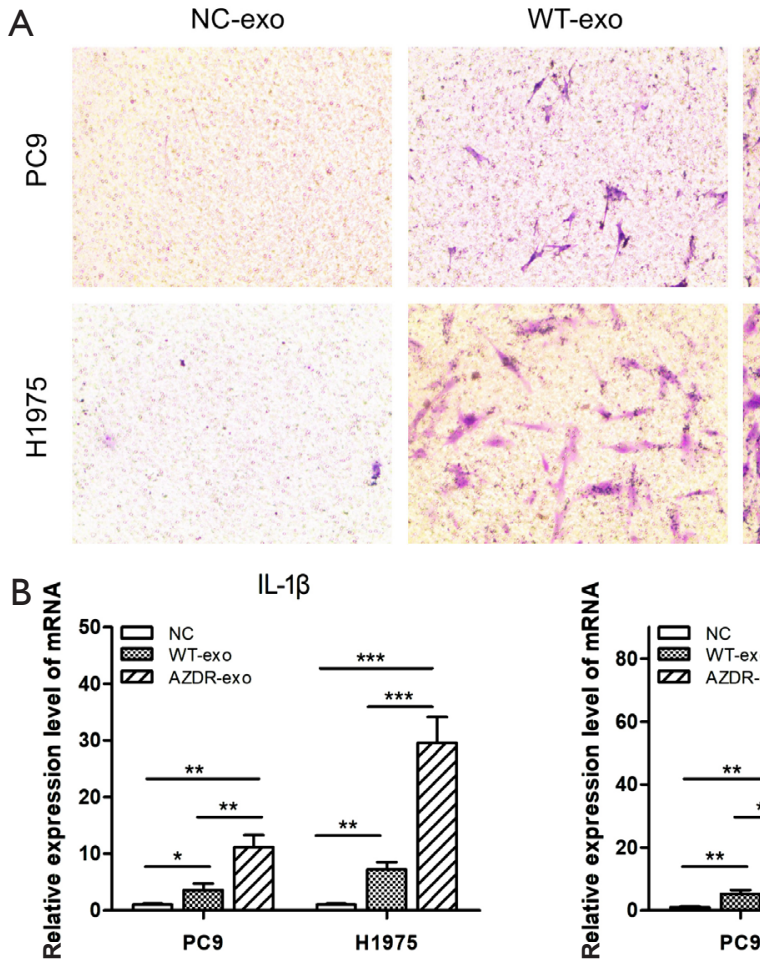

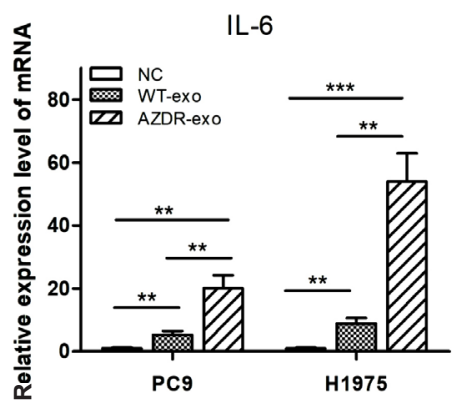

AZDR-exo
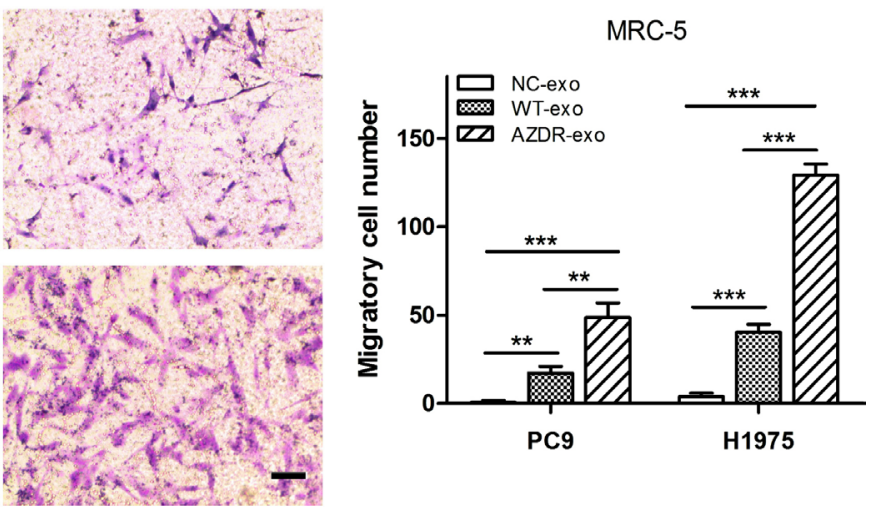

IL-8

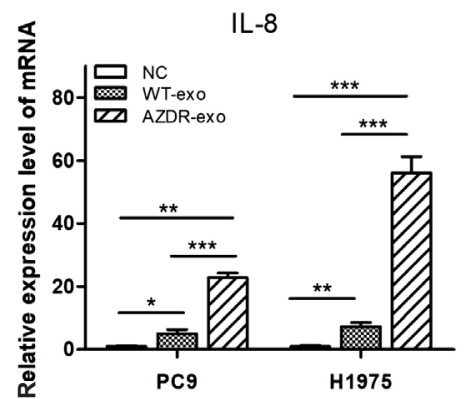

Figure 3 Exosomes derived from wild type or AZD9291-resistant NSCLC cells promoted the migration and inflammation of MRC-5 cells. (A) Representative images and statistical analysis of transwell assays analysis of MRC-5 cells co-cultured with or without exosomes derived from wild type or AZD9291-resistant H1975/PC9 cells ( $\mathrm{n}=3$ ) stained by crystal violet. (B) The mRNA levels of IL-1 $\beta$, IL-6, and IL-8 in MRC-5 cells co-cultured with or without exosomes derived from wild type or AZD9291-resistant H1975/PC9 cells ( $\mathrm{n}=3$ ). A length of 50 $\mu$ m was shown by scale bar and statistical significance was distinguished with $\mathrm{P}$ value: * $\mathrm{P}<0.05 ;{ }^{* *}, \mathrm{P}<0.01$; *** $\mathrm{P}<0.001$. NSCLC, non-small cell lung cancer.

without exosomes derived from wild type or AZD9291resistant PC9/H1975 cells. Interestingly, we found that the administration of exosomes derived from both wild type and AZD9291-resistant PC9/H1975 cells could promote the migration of MRC-5 cells (Figure $3 A$ ), and exosomes derived from AZD9291-resistant PC9/H1975 cells had a more profound effect compared to that from the wild type (Figure 3A). Furthermore, we examined the mRNA levels of inflammatory cytokines in exosome co-cultured MRC5 cells. Consistently, exosomes derived from both wild type and AZD9291-resistant PC9/H1975 cells could increase the mRNA levels of inflammatory cytokines (IL-1 $\beta$, IL6 , and IL-8) of MRC-5 cells, and the exosomes derived from AZD9291-resistant PC9/H1975 cells had a more profound effect compared to those from the wild type (Figure 3B). These results indicated that exosomes secreted by AZD9291-resistant NSCLC cells were more capable of promoting migration and inflammation of MRC-5 cells.

\section{Exosomal lnc-MZT2A-5:1 promoted the migration and inflammation of MRC-5 cells}

We further explored the molecular mechanisms underlying the activation of MRC-5 cells by ADZ9291resistant NSCLS cell derived exosomes. Given that exosomes encapsulate abundant lncRNAs and affect the communication between cells (21), we wanted to know whether lncRNAs in exosomes were involved in the effect of ADZ9291-resistant NSCLS cells on MRC-5 cells. Therefore, we performed lncRNA microarray analysis on the exosomes derived from wild type or ADZ9291resistant NSCLS cells (Figure 4A). We identified a total of 80 significantly up-regulated and 79 significantly downregulated lncRNAs (FC abs $\geq 2$ ), and the top $30 \operatorname{lncRNAs}$ 

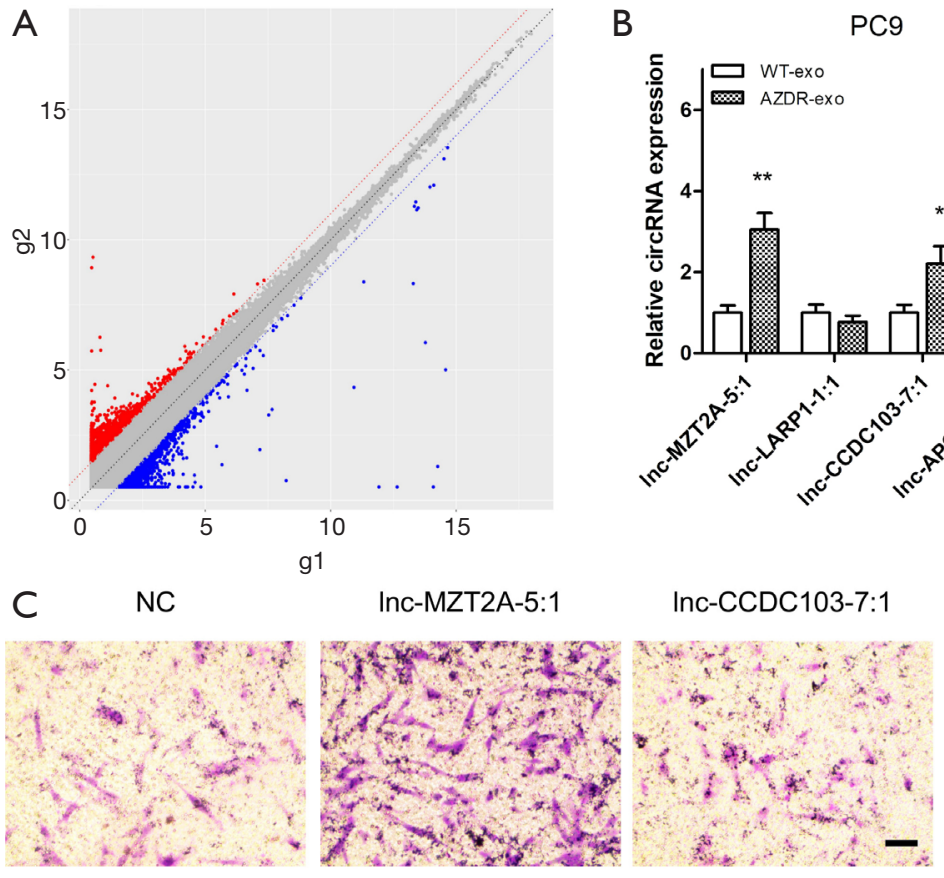

B

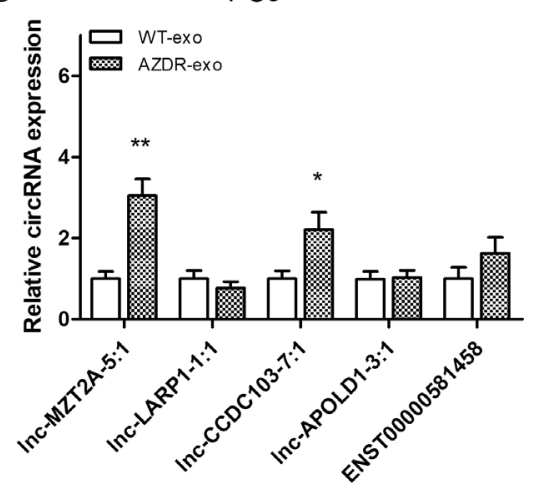

$\mathrm{H} 1975$
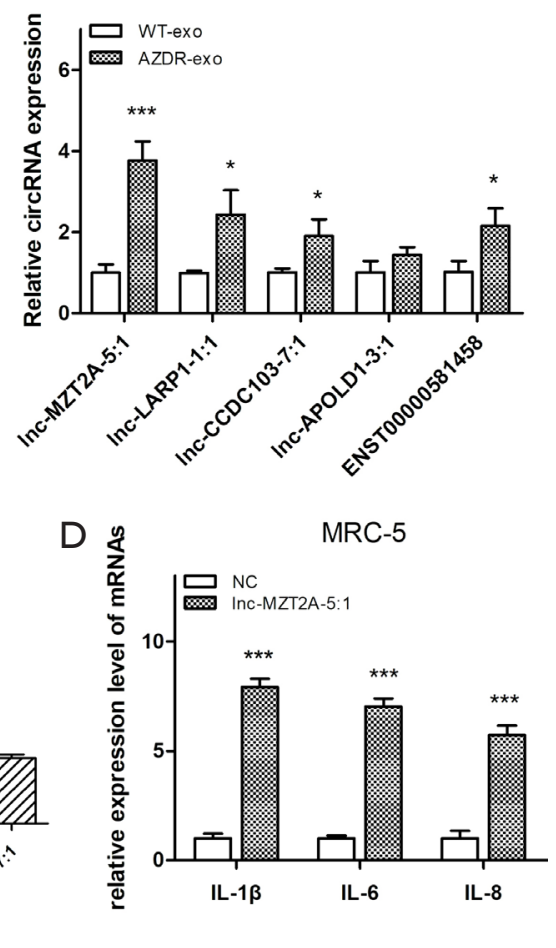

Figure 4 Exosomal lnc-MZT2A-5:1 promoted the migration and inflammation of MRC-5 cells. (A) Scatter plot of differentially expressed lncRNAs in exosomes derived from wild type and AZD9291-resistant NSCLC cells stained by crystal violet (red dot: significantly increased; blue dot: significantly decreased). (B) The expression levels of five candidate lncRNAs in exosomes derived from wild type or AZD9291resistant NSCLC cells. (C) Representative images and statistical analysis of transwell assays analysis of MRC-5 cells overexpressed with or without lnc-MZT2A-5:1 or lnc-CCDC103-7:1 (n=3), stained by crystal violet. (D) The mRNA levels of IL-1 $\beta$, IL-6, and IL-8 in MRC-5 cells overexpressed with or without lnc-MZT2A-5:1 ( $n=3)$. A length of $50 \mu \mathrm{m}$ was shown by scale bar and statistical significance was distinguished with $\mathrm{P}$ value: ${ }^{*}, \mathrm{P}<0.05$; ${ }^{* *}, \mathrm{P}<0.01$; ${ }^{* *}, \mathrm{P}<0.001$. NSCLC, non-small cell lung cancer.

with the highest FC abs are shown in Table 1. IncRNAs are considered correlated with either neighboring (cis) or distal (trans) protein-coding genes (22). We predicted the cis- and trans-target genes of the dysregulated lncRNAs, and GO enrichment and KEGG pathway analyses were performed by DAVID on these target genes. As shown in Table 2, part of the target genes are enriched in the pathways of NSCLC and small cell lung cancer. We speculated that certain IncRNAs are likely to regulate the expression of their target genes and participated in regulation of the malignant progression of lung cancer. The corresponding 15 lncRNAs which may be involved in the malignant progression of lung cancer are shown in Table 3. Furthermore, we found that five of the $15 \operatorname{lncRNAs}$ were also among the top 30 changed lncRNAs, as shown in Table 1. Therefore, we selected the five lncRNAs (lnc-MZT2A-5:1, lncLARP1-1:1, lnc-CCDC103-7:1, lnc-APOLD1-3:1, and
ENST00000581458), which are most likely to be involved in the progression of lung cancer, for further study.

Interestingly, our qRT-PCR results showed that only lnc-MZT2A-5:1 and lnc-CCDC103-7:1 were significantly increased in exosomes derived from ADZ9291-resistant PC9 and H1975 cells compared to the corresponding wild type (Figure 4B). Next, we overexpressed lnc-MZT2A-5:1 and lnc-CCDC103-7:1 in MRC-5 cells and found that the overexpression of lnc-MZT2a-5:1 but not lncCCDC103-7:1 could significantly promote the migration of MRC-5 cells through transwell assay (Figure 4C). Accordingly, overexpression of lnc-MZT2a-5:1 could also increase the mRNA levels of IL-1 $\beta$, IL-6, and IL8 , suggesting the increase of inflammation in MRC-5 cells (Figure 4D). These data indicated that exosomal lncMZT2a-5:1 may be involved in the malignant progression of NSCLC caused by ADZ9291-resistant. 
Table 1 Top 30 dysregulated exosomal lncRNAs in resistant cells

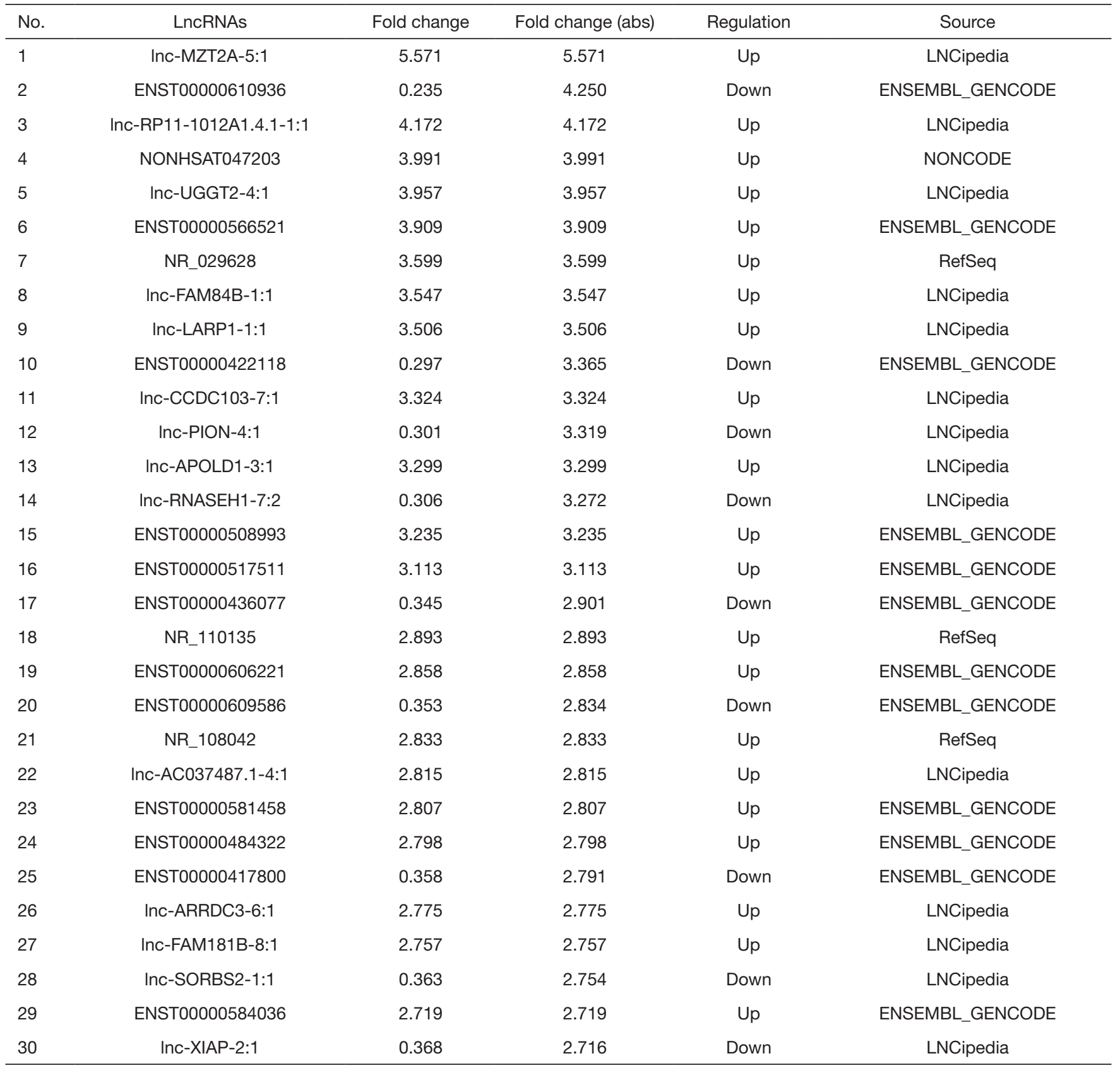

Table 2 Lung cancer-related pathways and target genes

\begin{tabular}{lcccc}
\hline Pathway ID & Description & Count & Target genes & Target types \\
\hline hsa05223 & Non-small cell lung cancer & 1 & FHIT & Cis-targets \\
hsa05222 & Small cell lung cancer & 1 & FHIT \\
hsa05222 & Small cell lung cancer & 8 & E2F2, CDK4, XIAP, APAF1, PIAS2, PIK3CD, SKP2, CYCS & Trans-targets \\
hsa05223 & Non-small cell lung cancer & 5 & E2F2, PRKCA, CDK4, PIK3CD, STK4 \\
\hline
\end{tabular}


Table 315 candidate lncRNAs

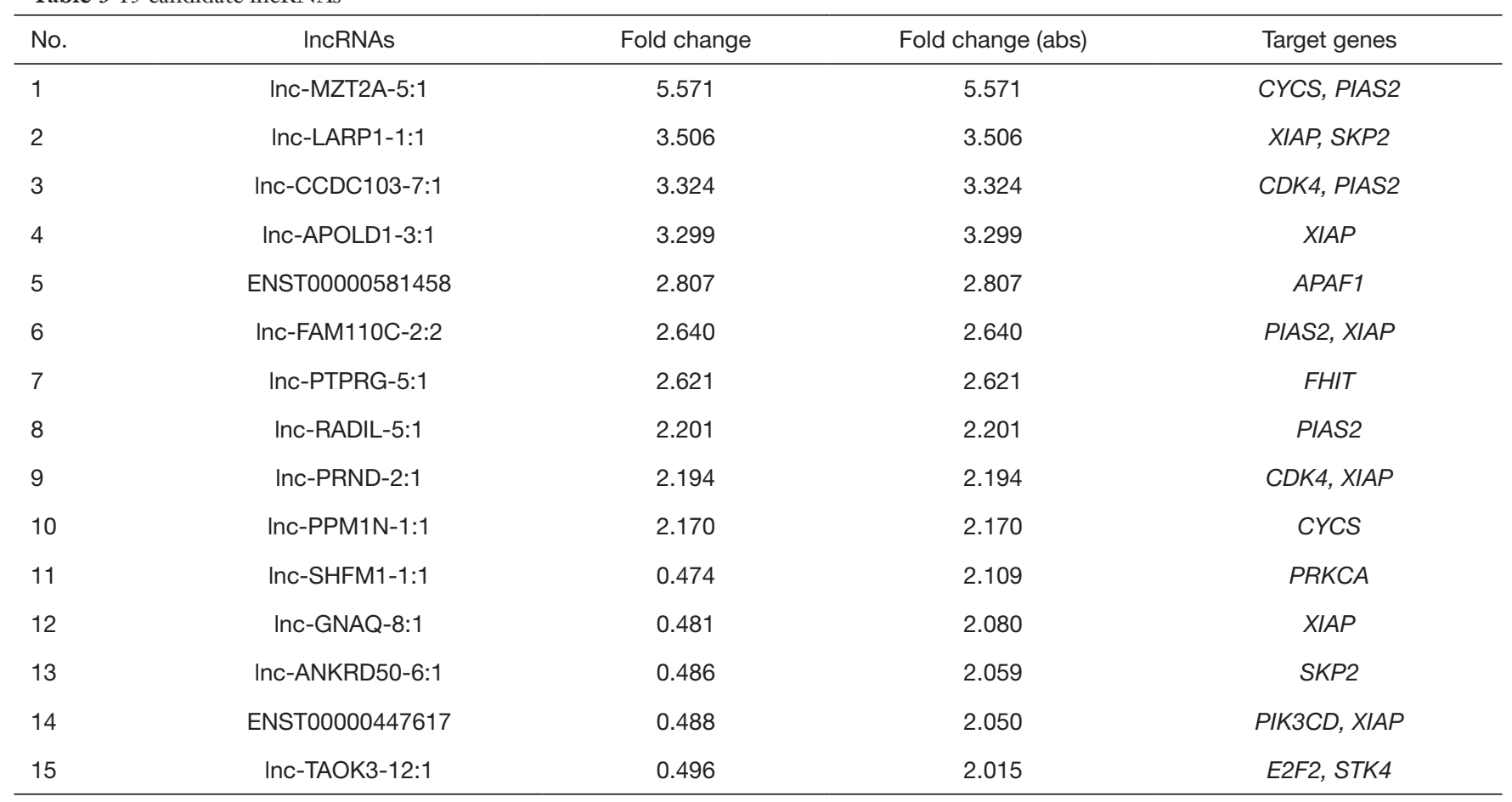

\section{Discussion}

Ahe platinum-based dual-drug combination dosing regimen has become the treatment of choice for advanced NSCLC. With in-depth study of the molecular mechanism of tumorigenesis, molecular targeted therapy such as EGFRTKIs has made breakthrough progress in controlling the progression of advanced NSCLC (23). Osimertinib has been widely used in the clinical treatment of NSCLC with T790M mutation, but the occurrence of drug resistance affects its long-term efficacy. Therefore, the exploration of osimertinib resistance mechanisms in NSCLC has become an important focus of research in the field. Here, we found that the supernatant and exosomes derived from wild type and AZD9291-resistant H1975/PC9 cells could promote the migration of MRC-5 cells. We also identified the differentially expressed exosomal lncRNAs by microarray and found that lnc-MZT2A-5:1 could significantly promote the migration and inflammation of MRC- 5 cells. Our study identified new mechanisms of AZD9291 resistance and provided new potential targets for the treatment of NSCLC.

The TME is composed of ECM proteins, cytokines, microvesicles, and stromal cells and plays a crucial role in regulating drug resistance in NSCLC (21). As key mediators of intercellular information transmission, exosomes show promising potential in the sensitivity of tumor cells to drugs. Previous studies reported that exosomal H19 facilitated erlotinib resistance in NSCLC via the miR-615-3p/ATG7 axis (24), and that exosomal lncRNA urothelial carcinomaassociated 1 (UCA1) was involved in gefitinib resistance in NSCLC (25). Additionally, Deng et al. reported the molecular mechanisms of osimertinib resistance in resistant and sensitive H1975 cells (26). However, few studies have reported the relationship of exosomal lncRNAs between osimertinib resistant NSCLC cells and MRC-5 cells. In the present study, we identified that exosomal lnc-MZT2A-5:1 was significantly increased in exosomes derived from AZD9291-resistant NSCLC cells compared to that derived from wild type cells and could promote the migration and inflammation of MRC-5 cells, providing potential therapeutic targets for NSCLC.

The ECM is a complex network composed of elastic fibers, collagen, and non-collagen glycoproteins, and is the microenvironment for cell growth. Activated fibroblasts can secrete large amounts of inflammatory factors and participate in tumor progression, and can promote drug resistance in gastric cancer (27), esophageal cancer (28), prostate cancer (29), and tongue cancer (30). However, there are few studies reporting the relationship between 
inflammation and drug resistance in lung cancer. Our research shows that AZD9291-resistant NSCLC cellsderived exosomes can promote the migration and inflammation of MRC-5 cells. Importantly, the exosomes derived from wild type NSCLC could also promote the migration and inflammation of MRC-5 cells, although this had a weaker influence compared to that derived from AZD9291-resistant NSCLC cells. These data provide a new molecular mechanism for the rapid development of drug resistance in lung cancer.

Previous studies have shown that after cisplatin treatment, lung cancer cells secreted more exosomes that can carry miRNAs, which may in turn confer resistance to the cells (31). There are also many studies showing that exosomes may change the drug sensitivity of tumors by transferring miRNA $(32,33)$. However, there are few studies on lncRNA in drug-resistant cell-derived exosomes. To further study the specific mechanism of AZD9291-resistant NSCLC cell-derived exosomes to promote the progression of NSCLC, we performed microarray analysis of lncRNAs in exosomes derived from wild type and AZD9291-resistant NSCLC cells, and identified the exosomal lnc-MZT2A-5:1 was significantly increased and could promote the migration and inflammation of MRC-5 cells. Although the molecular mechanism underlying the regulation of lnc-MZT2A-5:1 on MRC-5 cells is still unclear, our prediction of cisand trans-target genes related to differentially expressed lncRNAs provides an idea for further exploration. For example, growing evidence suggests that FHIT, a cis-targets of dysregulated lncRNAs, is a candidate tumor suppressor for NSCLC (34). Moreover, CYCS, a predicted target gene of lnc-MZT2A-5:1, was reported to be involved in the regulation of autophagy and apoptosis of NSCLC cells (35). Therefore, based on the predicted target genes, we will further explore the molecular mechanisms underlying the exosomal lncRNAs regulating the biological functions of MRC-5 cells in the future.

In conclusion, our results indicate that exosomes derived from wild type and AZD9291-resistant NSCLC cells can promote the malignant phenotype of MRC-5 cells. More importantly, microarray was performed to analyze exosomal lncRNAs, and showed that lnc-MZT2A-5:1 was significantly up-regulated in the exosomes derived from AZD9291-resistant NSCLC cells and could promoted the activation and migration of MRC-5 cells compared to that derived from wild type NSCLC cells. Our study identified new mechanisms of AZD9291 resistance and provided new potential targets for the treatment of NSCLC.

\section{Acknowledgments}

Funding: This project was supported by the National Natural Science Foundation of China (NSFC82002427), Shanghai Chest Hospital Outstanding Talent Cultivation Project (2018YNZYB03).

\section{Footnote}

Reporting Checklist: The authors have completed the MDAR reporting checklist. Available at https://dx.doi. org/10.21037/atm-21-5186

Data Sharing Statement: Available at https://dx.doi. org/10.21037/atm-21-5186

Conflicts of Interest: All authors have completed the ICMJE uniform disclosure form (available at https://dx.doi. org/10.21037/atm-21-5186). The authors have no conflicts of interest to declare.

Etbical Statement: The authors are accountable for all aspects of the work in ensuring that questions related to the accuracy or integrity of any part of the work are appropriately investigated and resolved.

Open Access Statement: This is an Open Access article distributed in accordance with the Creative Commons Attribution-NonCommercial-NoDerivs 4.0 International License (CC BY-NC-ND 4.0), which permits the noncommercial replication and distribution of the article with the strict proviso that no changes or edits are made and the original work is properly cited (including links to both the formal publication through the relevant DOI and the license). See: https://creativecommons.org/licenses/by-nc-nd/4.0/.

\section{References}

1. Hirsch FR, Scagliotti GV, Mulshine JL, et al. Lung cancer: current therapies and new targeted treatments. Lancet 2017;389:299-311.

2. Rajurkar S, Mambetsariev I, Pharaon R, et al. NonSmall Cell Lung Cancer from Genomics to Therapeutics: A Framework for Community Practice Integration to Arrive at Personalized Therapy Strategies. J Clin Med 2020;9:1870.

3. Terlizzi M, Colarusso C, Pinto A, et al. Drug resistance in non-small cell lung Cancer (NSCLC): Impact of genetic 


\section{Page 10 of 11}

and non-genetic alterations on therapeutic regimen and responsiveness. Pharmacol Ther 2019;202:140-8.

4. Forde PM, Kelly RJ, Brahmer JR. New strategies in lung cancer: translating immunotherapy into clinical practice. Clin Cancer Res 2014;20:1067-73.

5. Lindeman NI, Cagle PT, Beasley MB, et al. Molecular testing guideline for selection of lung cancer patients for EGFR and ALK tyrosine kinase inhibitors: guideline from the College of American Pathologists, International Association for the Study of Lung Cancer, and Association for Molecular Pathology. J Mol Diagn 2013;15:415-53.

6. Cross DA, Ashton SE, Ghiorghiu S, et al. AZD9291, an irreversible EGFR TKI, overcomes T790M-mediated resistance to EGFR inhibitors in lung cancer. Cancer Discov 2014;4:1046-61.

7. Leonetti A, Sharma S, Minari R, et al. Resistance mechanisms to osimertinib in EGFR-mutated non-small cell lung cancer. Br J Cancer 2019;121:725-37.

8. Andrews Wright NM, Goss GD. Third-generation epidermal growth factor receptor tyrosine kinase inhibitors for the treatment of non-small cell lung cancer. Transl Lung Cancer Res 2019;8:S247-64.

9. Xie F, Zhou X, Fang M, et al. Extracellular Vesicles in Cancer Immune Microenvironment and Cancer Immunotherapy. Adv Sci (Weinh) 2019;6:1901779.

10. EL Andaloussi S, Mäger I, Breakefield XO, et al. Extracellular vesicles: biology and emerging therapeutic opportunities. Nat Rev Drug Discov 2013;12:347-57.

11. Li Y, Yin Z, Fan J, et al. The roles of exosomal miRNAs and lncRNAs in lung diseases. Signal Transduct Target Ther 2019;4:47.

12. Mueller MM, Fusenig NE. Friends or foes - bipolar effects of the tumour stroma in cancer. Nat Rev Cancer 2004;4:839-49.

13. Bremnes RM, Dønnem T, Al-Saad S, et al. The role of tumor stroma in cancer progression and prognosis: emphasis on carcinoma-associated fibroblasts and nonsmall cell lung cancer. J Thorac Oncol 2011;6:209-17.

14. Liang G, Zhu Y, Ali DJ, et al. Engineered exosomes for targeted co-delivery of miR-21 inhibitor and chemotherapeutics to reverse drug resistance in colon cancer. J Nanobiotechnology 2020;18:10.

15. Yu Y, Abudula M, Li C, et al. Icotinib-resistant HCC827 cells produce exosomes with mRNA MET oncogenes and mediate the migration and invasion of NSCLC. Respir Res 2019;20:217.

16. Gan R, Yang Y, Yang X, et al. Downregulation of miR221/222 enhances sensitivity of breast cancer cells to
Song et al. Exosomal Inc-MZT2A-5:1 promotes fibroblasts activation

tamoxifen through upregulation of TIMP3. Cancer Gene Ther 2014;21:290-6.

17. Liu X, Jiang T, Li X, et al. Exosomes transmit T790M mutation-induced resistance in EGFR-mutant NSCLC by activating PI3K/AKT signalling pathway. J Cell Mol Med 2020;24:1529-40.

18. Yang C, Yang H, Liu J, et al. Focus on exosomes: novel pathogenic components of leukemia. Am J Cancer Res 2019;9:1815-29.

19. Chen T, Luo J, Gu Y, et al. Comprehensive analysis of circular RNA profiling in AZD9291-resistant non-small cell lung cancer cell lines. Thorac Cancer 2019;10:930-41.

20. Dennis G Jr, Sherman BT, Hosack DA, et al. DAVID: Database for Annotation, Visualization, and Integrated Discovery. Genome Biol 2003;4:P3.

21. Poulet C, Njock MS, Moermans C, et al. Exosomal Long Non-Coding RNAs in Lung Diseases. Int J Mol Sci 2020;21:3580.

22. Yan P, Luo S, Lu JY, et al. Cis- and trans-acting lncRNAs in pluripotency and reprogramming. Curr Opin Genet Dev 2017;46:170-8.

23. Zheng L, Wang Y, Xu Z, et al. Concurrent EGFR-TKI and Thoracic Radiotherapy as First-Line Treatment for Stage IV Non-Small Cell Lung Cancer Harboring EGFR Active Mutations. Oncologist 2019;24:1031-e612.

24. Pan R, Zhou H. Exosomal Transfer of lncRNA H19 Promotes Erlotinib Resistance in Non-Small Cell Lung Cancer via miR-615-3p/ATG7 Axis. Cancer Manag Res 2020;12:4283-97.

25. Chen X, Wang Z, Tong F, et al. lncRNA UCA1 Promotes Gefitinib Resistance as a ceRNA to Target FOSL2 by Sponging miR-143 in Non-small Cell Lung Cancer. Mol Ther Nucleic Acids 2020;19:643-53.

26. Deng Q, Fang Q, Xie B, et al. Exosomal long non-coding RNA MSTRG.292666.16 is associated with osimertinib (AZD9291) resistance in non-small cell lung cancer. Aging (Albany NY) 2020;12:8001-15.

27. Zhai J, Shen J, Xie G, et al. Cancer-associated fibroblastsderived IL-8 mediates resistance to cisplatin in human gastric cancer. Cancer Lett 2019;454:37-43.

28. Che Y, Wang J, Li Y, et al. Cisplatin-activated PAI1 secretion in the cancer-associated fibroblasts with paracrine effects promoting esophageal squamous cell carcinoma progression and causing chemoresistance. Cell Death Dis 2018;9:759.

29. Vancauwenberghe E, Noyer L, Derouiche S, et al. Activation of mutated TRPA1 ion channel by resveratrol in human prostate cancer associated fibroblasts (CAF). 
Mol Carcinog 2017;56:1851-67.

30. Liao JK, Zhou B, Zhuang XM, et al. Cancer-associated fibroblasts confer cisplatin resistance of tongue cancer via autophagy activation. Biomed Pharmacother 2018;97:1341-8.

31. Qin X, Yu S, Zhou L, et al. Cisplatin-resistant lung cancer cell-derived exosomes increase cisplatin resistance of recipient cells in exosomal miR-100-5p-dependent manner. Int J Nanomedicine 2017;12:3721-33.

32. Bach DH, Hong JY, Park HJ, et al. The role of exosomes and miRNAs in drug-resistance of cancer cells. Int J Cancer 2017;141:220-30.

33. Zheng P, Chen L, Yuan X, et al. Exosomal transfer of

Cite this article as: Song L, Qian G, Huang J, Chen T, Yang Y. AZD9291-resistant non-small cell lung cancer cell-derived exosomal lnc-MZT2A-5:1 induces the activation of fibroblasts. Ann Transl Med 2021;9(20):1593. doi: 10.21037/atm-21-5186 tumor-associated macrophage-derived miR-21 confers cisplatin resistance in gastric cancer cells. J Exp Clin Cancer Res 2017;36:53.

34. Yan $W, X u$ N, Han X, et al. The clinicopathological significance of FHIT hypermethylation in non-small cell lung cancer, a meta-analysis and literature review. Sci Rep 2016;6:19303.

35. Allavena G, Cuomo F, Baumgartner G, et al. Suppressed translation as a mechanism of initiation of CASP8 (caspase 8)-dependent apoptosis in autophagy-deficient NSCLC cells under nutrient limitation. Autophagy 2018;14:252-68.

(English Language Editor: B. Draper) 
Supplementary

Table S1 Primer sequences used for qRT-PCR

\begin{tabular}{lccc}
\hline No. & IncRNAs & Forward (5'-3') & Reverse (5'-3') \\
\hline 1 & Inc-MZT2A-5:1 & AAGACCAGCCTGACCAACAC & CTGCCACCATTTCCTTTGTT \\
2 & Inc-LARP1-1:1 & AGGGAGGATCTTGCATTCCT & AAGAACGAAAAGGGTGAGCA \\
3 & Inc-CCDC103-7:1 & GCCACAGAGCAGCCTAACAT & CAGCACCATCTCAGCAAGAA \\
4 & Inc-APOLD1-3:1 & GCCCATCCGAACAAGAACTA & CTACCAAACATTCCCCCTCA \\
5 & ENST00000581458 & GGCAAAACTCCCTCATCAGA & TGACCAGCACCTCCACATAA \\
\hline
\end{tabular}

